\title{
A case of severely comminuted femoral supracondylar fracture successfully treated with non-vascular fibular graft
}

\section{Introduction}

Management of severely comminuted supracondylar fracture of femur with large bone defect and intraarticular involvement is extremely challenging. Various surgical treatment strategies have been reported in the management of this complex injury with variable results.

Fracture reduction and maintaining the alignment and length of the femur with conservative treatment is extremely difficult due to the comminution. Articular incongruity will eventually result in osteoarthritis and knee stiffness. Complications associated with prolonged immobilization also make this treatment option undesirable. Hence, many surgical treatment options had been proposed over the years, including ilizarov external fixation and multiple types of internal fixation devices such as 95 degree angled blade plate, condylar buttress plate, dynamic condylar screw with 95 degree side plate, locking compression plate, and ante grade/retrograde intramedullary nails.

The purpose of this case report is to demonstrate that despite the severity of the injury, good union and functional outcome is achievable using the relatively simple technique of distal femur plating with locking plate augmented with autogenous non-vascularized fibula bone graft.

\section{Case Report}

We presented a case of a 20 -year-old man who had an alleged motor vehicle accident. He presented to the emergency department with pain and bleeding of the right thigh. On examination, Glasgow Coma Scale score was 15 of 15 . There was a deep laceration wound at the anterolateral aspect of his distal right thigh measuring $8 \mathrm{~cm}$ by $4 \mathrm{~cm}$. There was deformity, local tenderness, and abnormal mobility in the distal part of the thigh but no distal neurovascular deficit. Radiographs of the right femur revealed a severely comminuted supracondylar fracture with bone loss and intraarticular involvement (Figure 1A \& 1B). The fracture was classified as AO type 33-C3.

The patient underwent emergency wound debridement. Extruding bone pieces were removed with significant bone loss noted. Devitalized soft tissues and loose bone fragments without soft tissue attachments were excised. The wound was irrigated with copious amount of saline mixed with povidone iodine. The wound was loosely stitched with sutures spaced apart widely.

He was then put on proximal tibial skeletal traction with 5 $\mathrm{kg}$ of weight. Intravenous antibiotics were given (cefuroxime, metronidazole and gentamycin) and wound dressings were done daily. The second stage of the surgery was done 1 week after the first. After weighing the different surgical options, we decided to proceed with internal fixation of the distal femur with a locking plate and ipsilateral autogenous fibular bone graft to bridge the bone defect.
Volume 10 Issue 2 - 2018

Kamarul Izham Kamarudin, Chen Boon Jein, Suresh Chopra

Department of Orthopaedic, Hospital Sultanah Bahiyyah Alor Setar, Malaysia

Correspondence: Kamarul Izham Kamarudin, Orthopaedic Specialist, Science University of Malaysia (USM M), Department of Orthopaedic, Hospital Sultanah Bahiyyah, KM 6 Jalan Langgar, 05460 Alor Setar, Kedah, Malaysia, Tel +6047406233, Email drizham75@gmail.com

Received: March 30, 2018 | Published: April 20, 2018

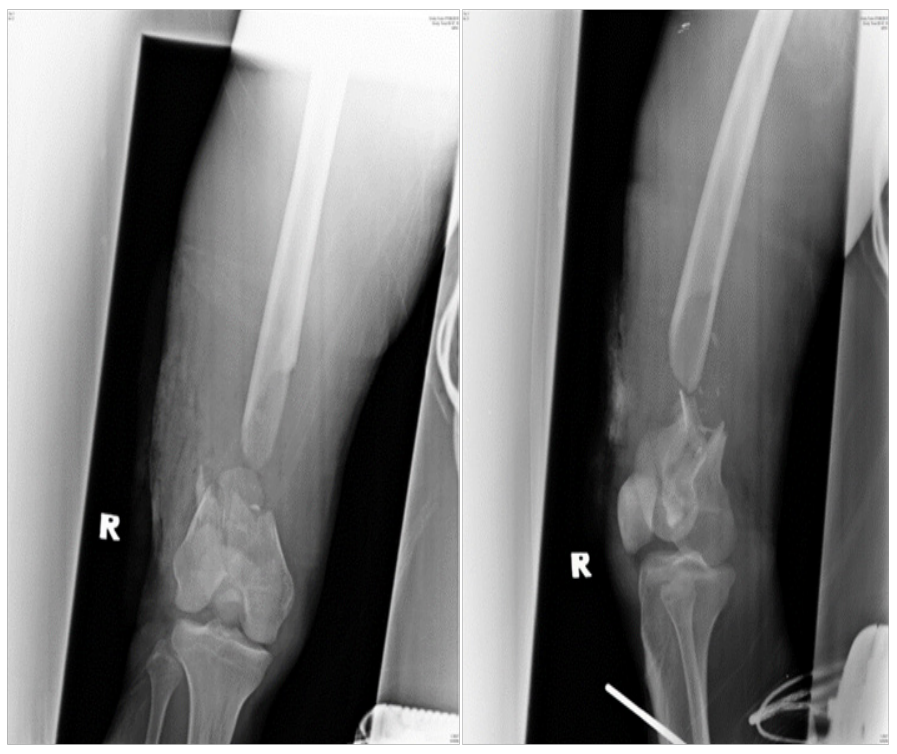

Figure I A (Left) Preoperative radiograph of the right knee,AP view, showed supracondylar fracture of right femur with bone loss and intraarticular involvement.

Figure I B (Right) Preoperative radiograph of the right knee, lateral view.

Two lateral incisions of 2 to $3 \mathrm{~cm}$ in length $10 \mathrm{~cm}$ apart were made at the proximal and distal end of the proposed length of the graft at the posterior margin of the fibula. Plane was developed between the personeal muscles and soleus muscles. Periosteum elevator was used to free the fibula anterior, lateral and posterior surfaces from tissue attachments. The proximal and distal end of the proposed fibula graft was then osteotomized. The graft was grasped with clamps 
and rotated externally along its axis to facilitate the gradual release of the fibula graft from its interosseous membrane attachment. Nonvascularized fibular graft of $9 \mathrm{~cm}$ in length was harvested from the ipsilateral leg. The graft was inserted into the medullary canal of the femur both proximally and distally to bride the bony defect. The fibular graft was secured to the femoral shaft proximally by a screw. Distal femur locking plate was then applied and secured proximally and distally to bridge the fracture site and the graft. Care was taken to prevent the displacement of the femoral shaft laterally which could alter the coronal plane alignment. The wound was closed in layers over a vacuum drain which was removed on post-operative day 3 .

Postoperative radiograph showed acceptable fracture alignment with autogenous fibular graft inside the medullary canal (Figures 2A $\& 2 B)$.

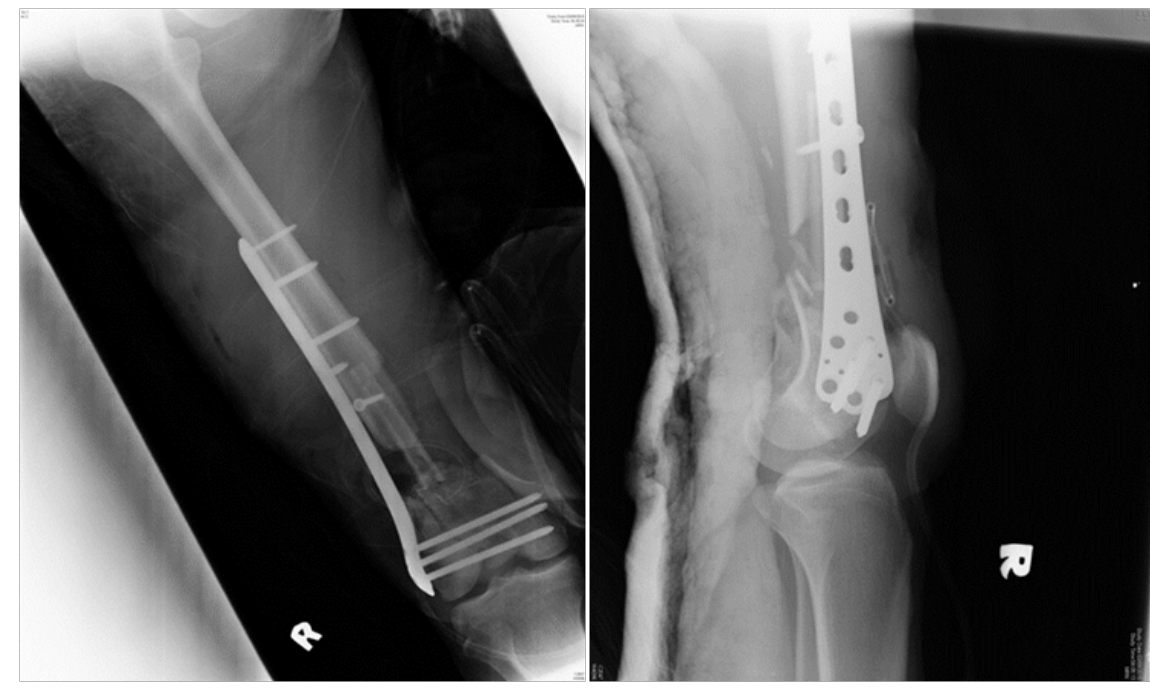

Figure 2A(Left) Immediate postoperative radiograph of the right femur, AP view, demonstrating good coronal alignment.

Figure 2B(Right) Immediate postoperative radiograph of the right knee, lateral view, demonstrating good sagittal alignment.

The patient was allowed active mobilization of the knee joint after surgery but weight bearing on the injured limb was strictly not allowed. The patient was allowed partial weight bear and full weight bear at 4 and 6 months respectively. The fracture achieved radiological union at 6 months after internal fixation and bone grafting (Figure $3 \mathrm{~A}$ \& 3B). Patient was able to ambulate independently without aid. Right lower limb however was shorter by $2 \mathrm{~cm}$ but patient did not have any functional complain and this can be equalized with a shoe lift. Right thigh muscles were atrophic. Passive range of motion of the right knee was 0 to 130 degrees while active range of motion was 10 to 120 degrees. Ankle joint was stable. Fibular graft donor site healed with no complication.
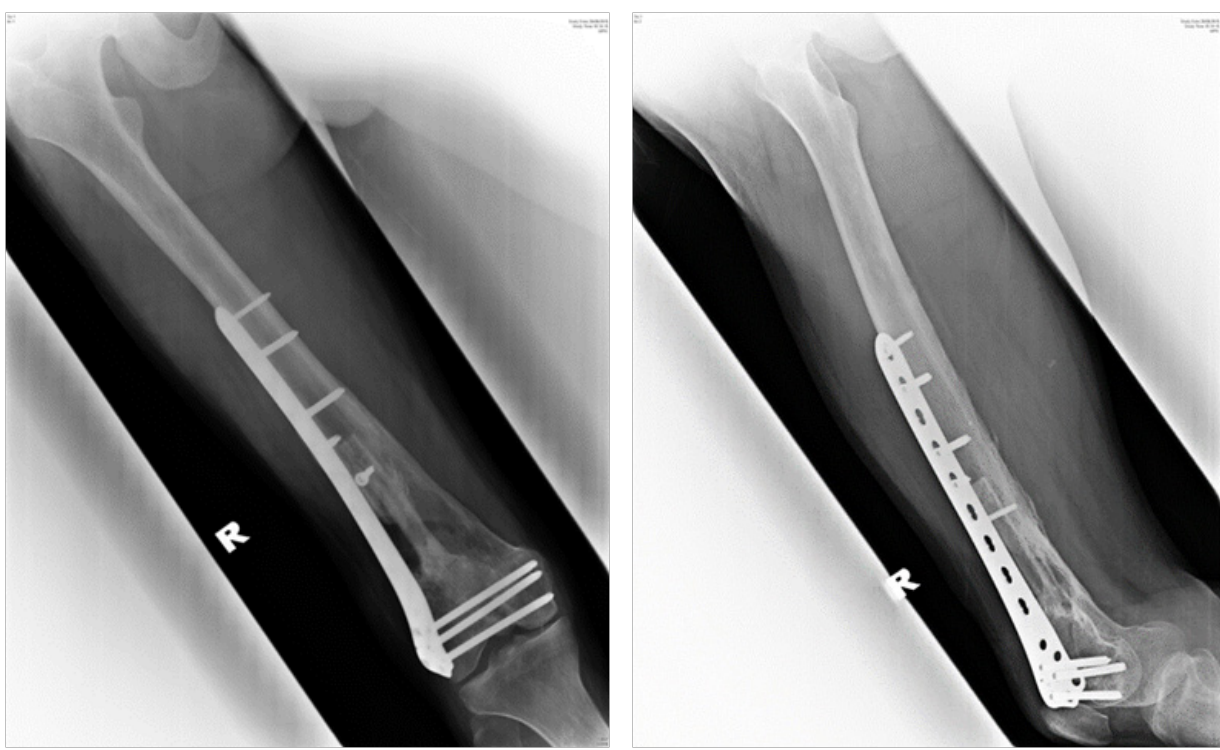

Figure 3A(Right) Radiograph of the right femur, AP view, six months after surgery showing fracture site union with incorporation of the fibula graft. Figure 3b (Left) Radiograph of the right femur, lateral view, six months after surgery showing fracture site union with incorporation of the fibula graft. 


\section{Case discussion}

Treatment of comminuted distal femur fractures is extremely challenging. The high energy trauma often results in very unstable fracture with comminution and bone loss. The associated articular and soft tissue injury makes it more difficult to treat. Problems commonly encountered are poor alignment, non-anatomical reduction, nonunion, malunion, limb length discrepancy and knee stiffness.

Retrograde femoral nail can be used in extraarticuar fracture, or simple intra-articular fractures with minimal displacement, but is not suitable for types $\mathrm{C} 2$ and $\mathrm{C} 3$ of the AO/ASIF system due to the location of the fracture and the degree of comminution and bone loss. It will not be able to provide adequate stability and maintain the fracture alignment.

Alternative surgical options include ilizarov external fixation in combination with autogenous fibular bone graft. Achieving and maintaining good fracture reduction and alignment is technically difficult. There are also risks of loss of reduction, septic arthritis, and pin tract infection. The range of motion of the knee will also be restricted due to tethering of the quadriceps muscles. Ramesh et al reported a case series involving 13 patients with $\mathrm{C} 3$ distal femur fractures treated with ilizarov ring fixator with ipsilateral autogenous fibular bone grafting. Fibula strut grafts were placed in the medullary canal of the femurs to bridge the fracture sites. Average time to union was 19 weeks. The mean range of motion of the knees was 77 degrees. 10 patients had good outcomes while the remaining 3 were poor due to constant pain, limitation of function and working capacity. 5 patients had pin-tract infections which were treated with antibiotics and dressings. ${ }^{1}$ Arazi et al reported the utilization of Ilizarov external fixation in 14 patients with severely comminuted supracondylar fractures of the distal femur: three type-A3, two type-C2 and nine type-C3 fractures (AO/ASIF system). All but one fractures united at a mean of 16 weeks. One third had fair to poor results while $21 \%$ had pin site infections. ${ }^{2}$

Double plating with bone grafting is another option. However, this surgical technique requires extensive soft tissue stripping on both sides of the femur, potentially reducing the blood supply and increasing the risk of nonunion and implant failure. Sanders et al treated 9 patients who sustained complex fractures of the distal femur with double plates and bone grafting to maintain reduction and alignment. All fractures eventually unite. Five patients had good functional outcome while the remaining four had only fair results. All patients had knee stiffness with less than 100 degrees of flexion. Knee flexion was less than 90 degrees in three patients. ${ }^{3}$ Ayman et al treated 12 patients with closed comminuted distal femur fractures using double plates and autogenous iliac bone grafting. 7 patients had satisfactory outcome while the remaining 5 patients had poor outcomes. Factors contributing to poor results were knee stiffness, pain and walking disability. ${ }^{4}$

Internal fixation with distal femoral locking plate was favored for its ability to provide immediate stability, allowing commencement of knee joint movement post operatively, thus reducing the risk of knee stiffness and muscular atrophy. Locking plate can also maintain the femoral length and at the same time protect the fibula graft to facilitate bone union.

Fibular bone grafting was indicated in this case in view that the bone defect was greater than $6 \mathrm{~cm}$ in length. Fibular bone graft was chosen for its biomechanical strength, and the significant length that could be harvested. Alternative bone graft options include allograft and synthetic bone graft, both of which eliminate bone harvesting related morbidities and complications. However, these bone grafts do not have osteogenic properties and incur high financial cost. In this case $\mathrm{x}$ ray of the non-affected femur on the opposite side was not taken to determine its length. Instead we measured the non-affected side clinically preoperatively to determine its length as a guide for us to correct the length of the fractured side. Intraoperatively after restoring the length and fixing the supracondylar femur, we found out that there was a big gap of bone loss measuring $6 \mathrm{~cm}$ in size. We decided to harvest a fibular graft with a length of $9 \mathrm{~cm}$ so that we could slide it inside the medullary cavity proximally and distally for better fixation of the graft. There was no fixed rules and regulation on how long we were supposed to harvest. We based it only on the size of the gap and we harvested it longer so as to be able to get it fixed inside the medullary canal.

Vascularised fibula bone graft was reported to be safe and effective in the treatment of large femoral defects with success rate of bone union as high as $95 \%$. This technique requires longer operation time due to the need for special microsurgical techniques. However, nonvascularized fibular graft was chosen for this patient due to good reported outcomes despite its simplicity.

Literatures have been vague regarding the length of the fibular bone that can be harvested safely for grafting. Approximately $20 \mathrm{~cm}$ of fibular bone can be harvested from the average adult fibula length of $36 \mathrm{~cm}$. Fibula is also dispensable since it only supports $15 \%$ of the axial weight through ankle joint. Despite the long length available for harvesting, care must be taken not to perform osteotomy too near the fibula head or ankle joint. In this case, the fibula bone was osteotomized $8 \mathrm{~cm}$ from the head of fibula. Osteotomy within $6 \mathrm{~cm}$ of the fibular head risks injuring the peroneal nerve which winds around the neck of fibula from posterior to anterior with the deep peroneal nerve also lying in close proximity on the anterior cortex of the fibula for $3-4 \mathrm{~cm}$.

It is believed that fibular graft harvesting within $8-10 \mathrm{~cm}$ of the ankle joint will cause ankle instability due to risk of injury to the ankle syndesmosis. However, Pacelli et al conducted a biomechanical analysis study on eleven fresh, paired cadaveric legs and concluded that only $10 \%$ of the fibula was needed distally to maintain ankle stability, which places the fibular osteotomy site just proximal to the syndesmotic ligaments. ${ }^{5}$

Possible complications associated with fibula graft harvesting include peroneal vessels injury and extensor hallucis longus weakness and compartment syndrome. Extensor hallucis longus is innervated by a single branch from the deep peroneal nerve making it very vulnerable to weakness with denervation. Ankle joint pain after fibular strut graft harvesting had also been reported with a prevalence ranging from $10 \%$ to $40 \%$.

The fracture united by 6 months with good functional outcome and with no graft harvest related complication.

\section{Conclusion}

In conclusion, severely comminuted open supracondylar fracture of the femur with significant bone loss can be effectively managed with bridging locking plate and autogenous non-vascularised fibula bone graft. Non-vascularized fibular bone graft is a relatively simple and inexpensive procedure which does not require microsurgical expertise 
and facilities. Good outcomes in terms of bone union, satisfactory knee joint range of motion and minimal donor site morbidity can be achieved as long as the proper surgical principles are adhered to.

\section{Acknowledgements}

None.

\section{Conflict of interest}

Authors declare there are no conflicts in publishing the article.

\section{References}

1. Ramesh LJ, Rajkumar SA, Rajendra R, et al. Ilizarov ring fixation and fibular strut grafting for $\mathrm{C} 3$ distal femoral fractures. J Orthop Surg (Hong Kong). 2004;12(1):91-95.
2. Arazi M, Memik R, Ogün TC, et al. Ilizarov external fixation for severely comminuted supracondylar and intercondylar fractures of the distal femur. J Bone Joint Surg Br. 2001;83(5):663-667.

3. Sanders R, Swiontkowski M, Rosen H, et al. Double-plating of comminuted, unstable fractures of the distal part of the femur. $J$ Bone Joint Surg Am. 1991;73:341-6.

4. Ayman ESK, Mostafa AA. Highly unstable complex C3-type distal femur fracture: can double plating via a modified Olerud extensile approach be a standby solution? J Orthop Traumatol. 2012;13(4):179-188.

5. Pacelli LL, Gillard J, McLoughlin SE, et al. A biomechanical analysis of donor-site ankle instability following free fibular graft harvest. $J$ Bone Joint Surg Am. 2003;85:597-603. 